\title{
Traumatic Pseudoaneurysm of Internal Carotid Artery presenting as Intractable Epistaxis in a Case of Maxillary Fracture
}

\author{
${ }^{1}$ Dinesh Kumar, ${ }^{2}$ Gyana Ranjan Sahu, ${ }^{3}$ Ajay Kumar, ${ }^{4}$ KK Mukherjee, ${ }^{5}$ Vidya Rattan
}

\begin{abstract}
Traumatic pseudoaneurysm of internal carotid artery is a rare complication of injury after craniomaxillofacial trauma. Delayed recurrent epistaxis after head and facial trauma is the most distinctive manifestation of traumatic internal carotid artery pseudoaneurysm. Epistaxis due to pseudoaneurysm of internal carotid artery is difficult to control, and may lead to hemorrhagic shock or asphyxia and thus seriously threatens life. The purpose of this paper is to present a rare case of recurrent epistaxis secondary to ICA pseudoaneurysm following blunt maxillofacial trauma. Endovascular stenting was performed and the recurrent epistaxis was successfully arrested.
\end{abstract}

Keywords: Epistaxis, Pseudoaneurysm, Internal carotid artery, Endovascular stent.

How to cite this article: Kumar D, Sahu GR, Kumar A, Mukherjee KK , Rattan V. Traumatic Pseudoaneurysm of Internal Carotid Artery presenting as Intractable Epistaxis in a Case of Maxillary Fracture. J Postgrad Med Edu Res 2014;48(2):100-102.

\section{Source of support: Nil}

Conflict of interest: None

\section{INTRODUCTION}

Pseudoaneurysm, also known as a false aneurysm, is an out-pouching of a blood vessel, with actual disruption of one or more layers of its wall. ${ }^{1}$ Traumatic internal carotid artery (ICA) pseudoaneurysm is rare complication of blunt craniofacial trauma, with a reported incidence approximately $1 \%$ of all blunt trauma. ${ }^{2}$ Internal carotid artery pseudoaneurysm is a rare and potentially fatal cause of epistaxis that demands prompt recognition. Although a majority of ICA pseudoaneu-

\footnotetext{
${ }^{1}$ Senior Resident, ${ }^{2}$ Junior Resident, ${ }^{3}$ Assistant Professor,

${ }^{4,5}$ Additional Professor

$1,2,5$ Unit of Oral and Maxillofacial Surgery, Oral Health Sciences Centre, Postgraduate Institute of Medical Education and Research, Chandigarh, India

${ }^{3}$ Department of Radiology, Postgraduate Institute of Medical Education and Research, Chandigarh, India

${ }^{4}$ Department of Neurosurgery, Postgraduate Institute of Medical Education and Research, Chandigarh, India

Corresponding Author: Dinesh Kumar, Senior Resident Unit of Oral and Maxillofacial Surgery, Oral Health Sciences Centre, Postgraduate Institute of Medical Education and Research, Chandigarh, India, Phone: 0172-2756261, e-mail: dr_dinesh78@yahoo.com
}

rysms are associated with trauma or infection, cases associated with pregnancy, iatrogenic injury, tumor invasion, and radiotherapy have also been reported. Recurrent epistaxis following blunt craniofacial trauma should alert clinicians to the possibility of traumatic (ICA) pseudoaneurysm. Endovascular intervention of ICA pseudoaneurysm is the treatment of choice in managing such cases. The purpose of this paper is to present a rare case of recurrent epistaxis secondary to ICA pseudoaneurysm following blunt maxillofacial trauma.

\section{CASE REPORT}

A 21-years-old female suffered from injury due to road traffic accident in September 2013. She reported to the oral and maxillofacial surgery unit with complaints of swelling on face, inability to chew and restricted mouth opening. The patient reported oronasal bleeding and vomiting after trauma but had no history of unconsciousness and altered sensorium. On palpation, step and tenderness at left infraorbital rim and mobility at Le Fort-I level was present. Diagnosis of fracture left zygomatic complex and bilateral Le Fort-I maxilla was confirmed with a computed tomography (CT) scan of head and face (Figs 1A and B) with no other abnormal finding.

The patient underwent open reduction and internal fixation with titanium miniplates for the fractured maxilla under general anesthesia. She was discharged after 2 days without any complications. The patient was followed up on seventh day after operation and sutures were removed.

The patient reported on eleventh postoperative day with complaint of bleeding from left nostril and was admitted in hospital for observation. During the 2 days hospital stay, there was no episode of epistaxis and she was discharged. On fifteenth postoperative day, she reported again in the emergency unit with epistaxis. On endoscopic examination by ear, nose, throat surgeon no active bleeding point in the nasopharynx was detected. She was kept under observation for 3 days during which there was no episode of epistaxis and hence was discharged.

On 23rd postoperative day, the patient again reported with complaint of epistaxis. ACT angiogram was performed which revealed pseudoaneurysm of left internal carotid artery (Figs 2A and B). After a neurosurgery consultation, 

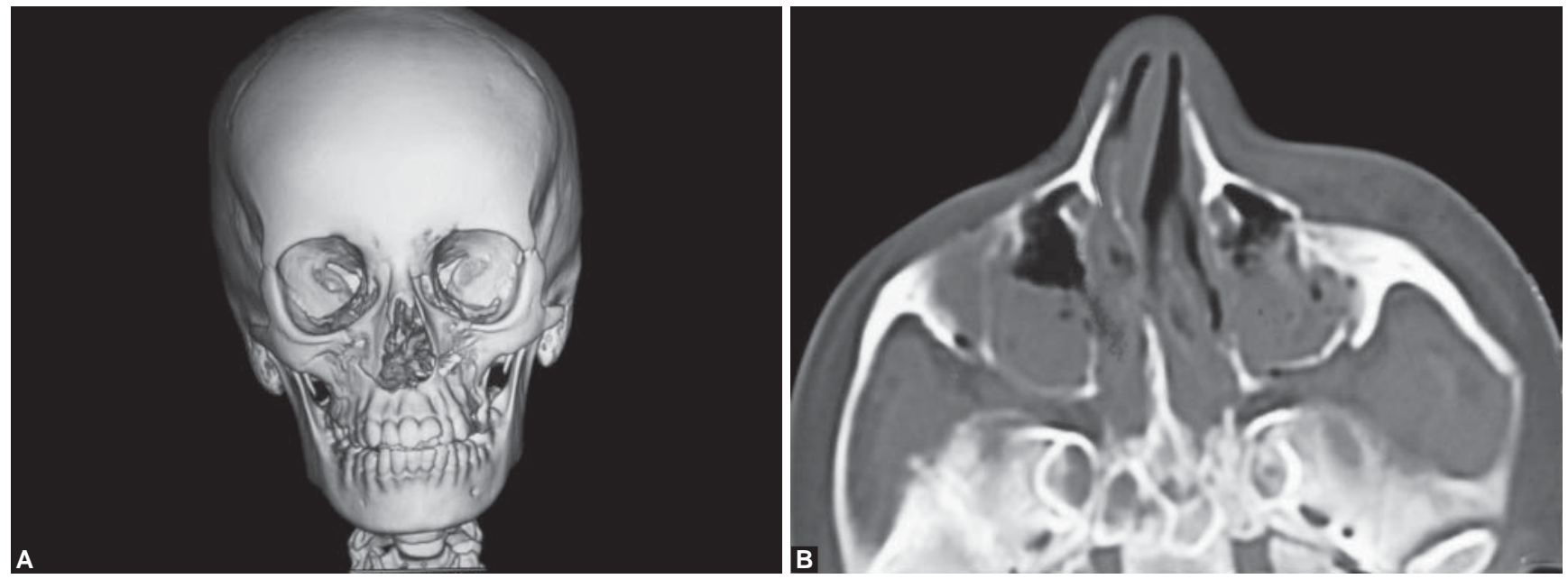

Figs $1 \mathrm{~A}$ and B: Computerized tomographic scan showing Le Fort-1 fracture of maxilla and inferiorly displaced left zygomatic complex: (A) $3 \mathrm{D}$ reconstruction and $(\mathrm{B})$ axial cut
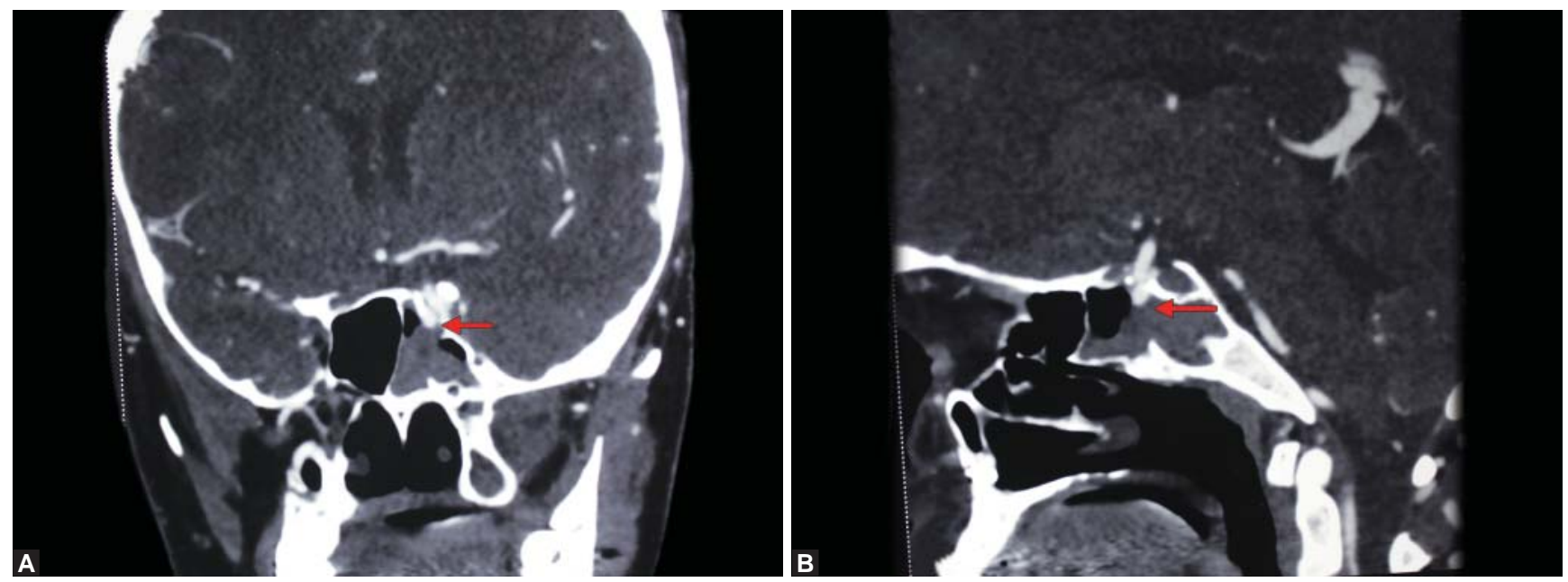

Figs 2A and B: Computed tomography angiography coronal and sagittal reformatted images show, pseudoaneurysms of left internal carotid artery arising from cavernous segment (arrows)

the intervention radiologist performed digital subtraction angiography of the carotid artery. Its findings confirmed that there were two traumatic pseudoaneurysm arising from cavernous segment (C4/C3 segment) of left internal carotid artery, larger measuring approximately $4.4 \times 3$ mm directed anteroinferiorly and smaller located postero-medially measuring approximately $3.5 \times 2 \mathrm{~mm}$ in size (Fig. 3).

Due to repeated epistaxis episodes the patient's hemoglobin level had reduced from 13.2 to $5.9 \mathrm{gm} / \mathrm{dl}$ and for the same 2 units of packed red cells were transfused. On 25th postoperative day, the left internal carotid artery pseudoaneurysm stenting was performed under general anesthesia using a coronary stent (Aneugraft, $3.5 \times 23 \mathrm{~mm}$.

ITGI Medical Ltd, Hailan St., Israel) (Fig. 4) without any intraoperative complication. Postoperatively the patient was put on oral anticoagulant therapy. Angiography done at one month post-stenting showed patency of the stent and nonopacification of pseudoaneurysm. The patient has been symptom free at her 6th months follow-up.

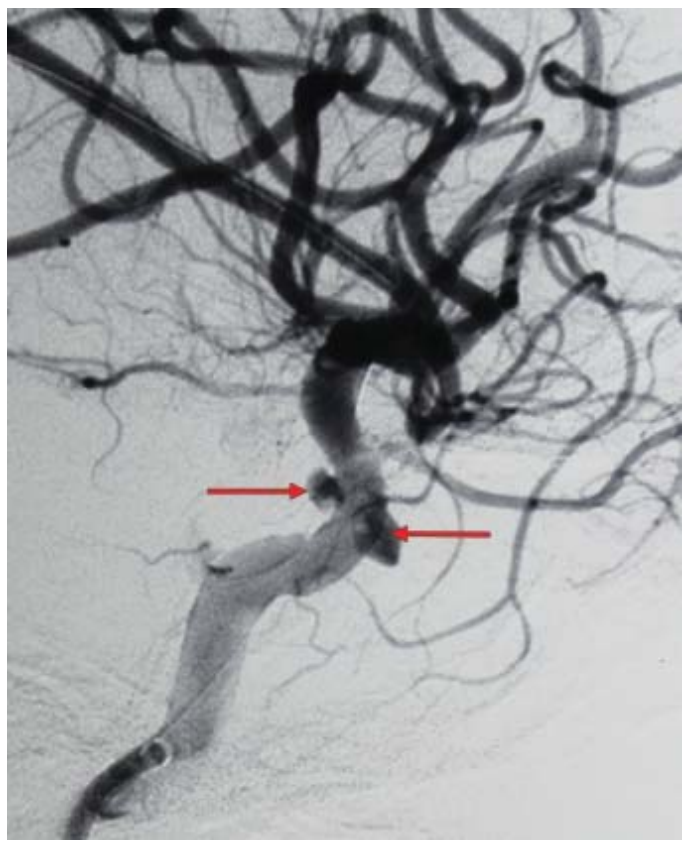

Fig. 3: The internal carotid angiogram reveals pseudoaneurysm (arrows) in the region of cavernous segment showing larger directed anteroinferiorly and smaller posteromedially 


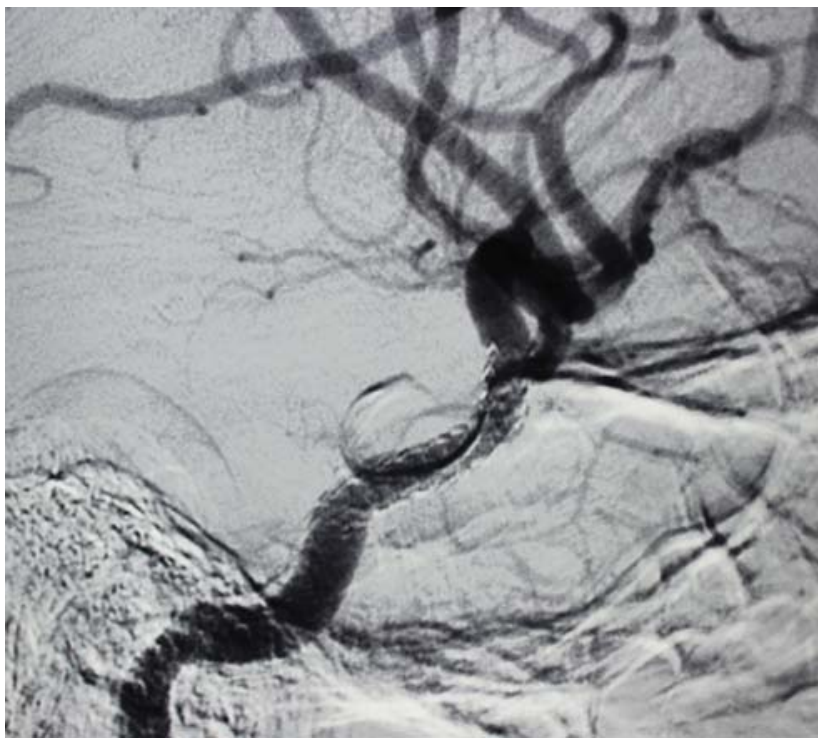

Fig. 4: Post-stent graft placement shows complete nonopacification of the pseudoaneurysm with patent internal carotid artery lumen

\section{DISCUSSION}

An aneurysm is an abnormal widening or ballooning of a portion of an artery due to weakness in the wall of the blood vessel. A true aneurysm is one that involves all three layers of the wall of an artery (media, intima and adventitia). A pseudoaneurysm or false aneurysm occurs as the result of a leaking hole in a blood vessel. Traumatic pseudoaneurysm of the ICA pathogenesis involves partial vessel transection and formation of a hematoma. The bleeding stops with counter pressure of the hematoma. The unclotted portion of the hematoma located around the injured wall is filled by circulating blood in continuity with the arterial lumen. The hematoma liquefies in around 1 week and may re-bleed. The blood causes an inflammatory reaction in surrounding tissue with the formation of a fibrous capsular wall and development of an epithelial lining. Continuous pulsatile forces can result in enlargement, weakening and breakdown of the fibrous wall, ${ }^{3-5}$ which cause repeated bleeding. Patients with traumatic internal carotid artery pseudoaneurysm present with repeated epistaxis that is difficult to control, a condition that often leads to hemorrhagic shock or asphyxia and thus seriously threatens life.

Pseudoaneurysms are uncommon in the maxillofacial region and usually result from blunt or penetrating injury, or surgical trauma. ${ }^{6}$ Iatrogenic pseudoaneurysm of ICA have been reported following maxillary osteotomies due to excessive force applied during mobilization of maxilla and fracture line traversing along the base of skull. The present patient possibly had developed pseudoaneurysm following road traffic trauma, as minimal manipulation was done during fixation of maxillary fracture, as it was minimally displaced. Traumatic pseudoaneurysms located near the skull base are hazardous to approach and challenging to repair. ${ }^{7}$ In the past, surgical treatment of ICA pseudoaneurysons has been the preferred approach; ${ }^{8,9}$ however, the close proximity to the skull base makes repair difficult. The development of endovascular techniques now provides a feasible alternative to direct surgical repair for vascular injuries of the ICA near the skull base. ${ }^{7,10}$ Our patient underwent a successful endovascular treatment of an ICA pseudoaneurysm.

\section{CONCLUSION}

Following blunt craniofacial trauma, delayed onset epistaxis should alert the clinician to possible traumatic internal carotid artery injury and angiography should be done to rule outpseudoaneurysm. Early diagnosis and treatment of ICA pseudoaneurysm is essential to minimize morbidity and mortality.

\section{REFERENCES}

1. Luo CB, Teng MM, Chang FC, Chang CY. Role of CT and endovascular embolization in managing pseudoaneurysms of the internal maxillary artery. J Chin Med Assoc 2006;69(7):310-316.

2. Tseng YY, Yang ST, Yeh YS, Yang TC, Wong HF. Traumatic internal carotid artery pseudoaneurysm mimicking sphenoid sinus tumor. Rhinology Dec 2007;45(4):332-334.

3. Celil G, Engin D, Orhan G, barbaros C, Hakan K, Adil E. Intractable epistaxis related to cavernous carotid artery pseudoaneurysm: treatment of a case with covered stent. Auris Nasus Larynx 2004;31:275-278.

4. Bavinzski G, Killer M, Knosp E, Ferraz-Leite H, Gruber A, Richling B. False aneurysm of the intracavernous carotid artery. Report of 7 cases. Acta Neurochir 1997;139:37-43.

5. Germiller JA, Myers LL, Harris MO, Bradford CR. Pseudoaneurysm of the proximal facial artery presenting as oropharyngeal hemorrhage. Head Neck 2001;23:259-263.

6. Pappa H, Richardson D, Niven S. False aneurysm of the facial artery as a complication of sagittal split osteotomy. J CranioMaxillofac Surg 2008;36:180.

7. Redekop G, Marotta T, Weill A. Treatment of traumatic aneurysms and arteriovenous fistulas of the skull base by using endovascular stents. J Neurosurg 2001;95:412.

8. Ramadan F, Rutledge R, Oller D, et al. Carotid artery trauma: a review of contemporary trauma center experiences. J Vas Surg 1995;21:46.

9. D’Alise MD, Vardiman AB, Kopitnik TA Jr, et al. External carotid to middle cerebral bypass in the treatment of complex internal carotid injury. J Trauma 1996;40:452.

10. Salgarelli A, Morana G, Beltramello A. Pseudoaneurysm of the lingual artery: A case report. J Oral Maxillofac Surg 1997; $55: 860$. 